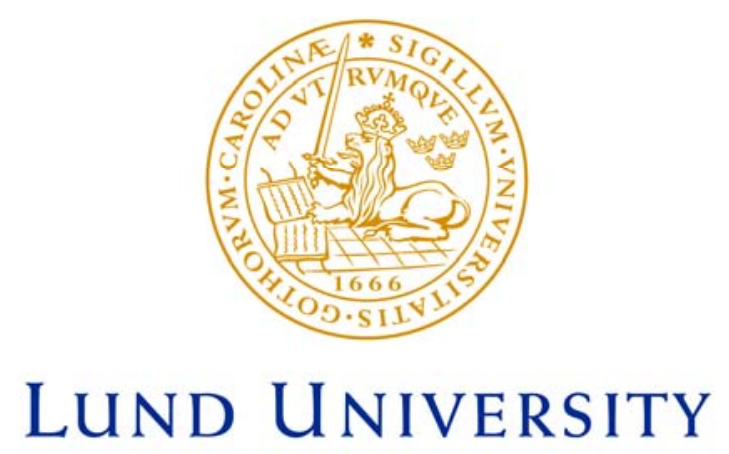

Faculty of Medicine

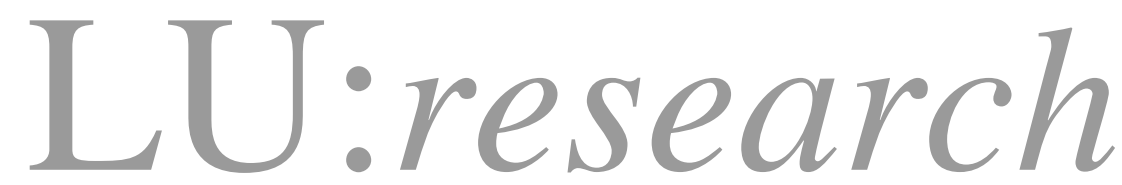

Institutional Repository of Lund University

This is an author produced version of a paper published in Thrombosis research. This paper has been peer-reviewed but does not include the final publisher proof-corrections or journal pagination.

Citation for the published paper:

Malm, Karl and Arnljots, Bjorn and Persson, Ing-Marie and Dahlback, Bjorn.

"Antithrombotic and anticoagulant effects of wild type and Gla-domain mutated human activated protein $\mathrm{C}$ in rats."

Thrombosis research, 2006, Issue: Dec 11.

http://dx.doi.org/10.1016/j.thromres.2006.11.004

Access to the published version may require journal subscription.

Published with permission from: Elsevier 


\section{Antithrombotic and Anticoagulant effects of Wild Type and Gla-Domain Mutated Human Activated Protein C in Rats}

Karl Malm, MD, PhD*; Björn Arnljots, MD, PhD*, Ing-Marie Persson**, Björn Dahlbäck, $\mathrm{MD}, \mathrm{PhD}^{* *}$

From the *Department of Clinical Sciences, Division of Reconstructive Surgery,

**Department of Laboratory Medicine, Division of Clinical Chemistry, University Hospital, Malmö, Sweden.

Word Count: 3944

Corresponding author:

Karl Malm

Department of Plastic and Reconstructive Surgery

University Hospital Malmö

SE-205 02 Malmö

Sweden

Telephone: +4640331000

Fax: +46403362 71

E-mail: karl.malm@med.lu.se 


\section{ABSTRACT}

The antithrombotic and anticoagulant effects of recombinant wild type (WT) and mutated human activated protein C (hAPC) were investigated using a rat model of arterial thrombosis. Recent in vitro studies using human plasma have shown enhanced anticoagulant effects of hAPC by mutagenesis of either loop 148 in the serine protease domain or of the Gla domain. The Gla-domain mutant QGNSEDY-hAPC (=H10Q/S11G/S12N/D23S/Q32E/N33D/H44Y) was found to be particularly active as an anticoagulant. We now combined the two mutations to create the variant QGNSEDY-hAPC:B148 and investigated the in vivo effects of this variant as well as of QGNSEDY-hAPC and WT hAPC using a rat model of arterial thrombosis. In vitro clotting experiments using rat plasma demonstrated WT hAPC to be inefficient, whereas both mutant hAPC variants yielded distinct dose dependent anticoagulant effects. In the arterial injury model, a segment of the left common carotid artery was opened longitudinally. An endarterectomy was performed and the arteriotomy was closed, whereafter the vessel was reperfused and the patency rate determined after 31 minutes. Three treatment groups each containing 10 rats and a control group of 20 animals were in a blind random fashion given intravenous bolus injections of $0.8 \mathrm{mg} / \mathrm{kg} \mathrm{WT}$ or mutant hAPC or vehicle only. The ex vivo clotting times of plasma drawn 3 minutes after the injections, as compared to baseline clotting times, were approximately doubled by QGNSEDY-hAPC and tripled by QGNSEDY-hAPC:B148 infusions, while WT APC had little effect. Compared to the control group, none of the hAPC preparations had significant antithrombotic effect or increased arteriotomy bleeding.

Keywords: arterial thrombosis, mutated activated protein C, bleeding, animal model 
Abbreviations: WT, wild type; APC, activated protein C; PS, protein S; EPCR, endothelial protein C receptor; PAR, protease activated receptor; APTT, activated partial thromboplastin time; PT, prothrombin time; TBS, tris-buffered saline; BSA, bovine serum albumin; SDSPAGE, sodium dodecyl sulfate polyacrylamid gel electrophoresis. 
Protein $\mathrm{C}$ is a vitamin K-dependent protein that circulates in plasma as a zymogen to a serine protease with anticoagulant properties [1-4]. In response to thrombin generation, protein C is activated on the endothelial surface by the thrombin-thrombomodulin complex [4]. Activated protein C (APC) together with its cofactor protein S (PS) effectively down-regulates blood coagulation by cleaving and inhibiting the two cofactors activated factor $\mathrm{V}$ (FVa) and activated factor VIII (FVIIIa) [5, 6]. Several cleavage sites have been identified in both FVa and FVIIIa, of which the cleavages at Arg306 and Arg506 in FVa have been studied in detail [7-12].

The physiological importance of the anticoagulant properties of APC is best illustrated by the increased risk of venous thrombosis in individuals with genetic defects affecting the protein $\mathrm{C}$ system $[1,13,14]$. In addition to its anticoagulant properties, APC has been shown to possess anti-inflammatory functions, which appear to depend on the presence of endothelial protein C receptor (EPCR) [15]. EPCR is suggested to direct the proteolytic activity of APC towards the protease activated receptor 1 (PAR1) rather than to FVa and FVIIIa [16]. APC has been shown to be efficient in the treatment of septic shock, the beneficial effects possibly being due to both the anticoagulant and anti-inflammatory properties of APC [15, 17].

The anticoagulant properties of APC make it an interesting therapeutic candidate for prevention and treatment of venous and arterial thrombosis. There are several reports on record investigating the effect of APC in different animal models [18-31]. We have previously described powerful antithrombotic effects of bovine APC (bAPC) in combination with bovine PS (bPS) in both rabbit and rat models of deep arterial injury [18-20, 32].

We have recently created several recombinant human protein $C$ variants with enhanced anticoagulant activity and the aim of the present investigation was to elucidate their 
antithrombotic potential in the arterial thrombosis model in the rat. In one of the variants, QGNSEDY-hAPC (H10Q/S11G/S12N/D23S/Q32E/N33D/H44Y), the membrane-binding properties of APC were altered by mutagenesis of the $\gamma$-carboxyglutamic acid (Gla)-rich domain. This variant proved to be highly effective as an anticoagulant in human plasma and QGNSEDY-hAPC alone was found to be more potent than WT hAPC together with hPS in a FVa degradation assay [33]. In another recombinant hAPC variant (hAPC:B148), loop 148 in the serine protease domain was exchanged for the corresponding bovine loop, which resulted in a hAPC variant with modestly enhanced catalytic and anticoagulant activity [34]. By combining the mutations in QGNSEDY-hAPC and hAPC:B148 we have now created a new hAPC variant (QGNSEDY-hAPC:B148), which is more effective as an anticoagulant than either of the two original variants.

In this study, we have investigated the antithrombotic, antihaemostatic, and anticoagulant effects of recombinant WT hAPC as well as the two recombinant hAPC variants QGNSEDYhAPC and QGNSEDY-hAPC:B148. Despite demonstrating strong anticoagulant effects in vitro using rat plasma, the two hAPC variants failed to demonstrate antithrombotic effects in the arterial thrombosis model in the rat. 


\section{MATERIALS AND METHODS}

\section{Recombinant protein $C$ variants}

Full length cDNAs for the QGNSEDY-hPC and hPC:B148 introduced in the HindIII and $\mathrm{XbaI}$ sites in the pRC/CMV vector were created with recombinant DNA-techniques, as previously described [33-36]. The mutations in the QGNSEDY variant was H10Q/S11G/S12N/D23S/Q32E/N33D/H44Y, where the first letter preceding the amino-acid number is the one-letter abbreviation for the wild-type amino acid, and the letter after the number refers to the amino acid residue introduced by the mutagenesis [33].

The amino-acid numbers refer to the positions in the mature protein, i.e. after removal of the signal sequence and propeptide. In the hPC: B148 mutant, the 148 loop in human protein C, GWGYHSSREKEAKRN (the underlined amino acids correspond to positions 303-310 in the linear sequence, and to $144-149 \mathrm{~b}$ according to the chymotrypsinogen numbering), was replaced by the four residues shorter corresponding loop in bAPC (GWGYRDETKRN) [34]. The cDNA for the new mutant used in this study QGNSEDY-hPC:B148 was constructed by combining the appropriate fragments of the cDNAs for QGNSEDY and hPC: B148. Thus, the 360 bp HindII-SalI fragment of QGNSEDY cDNA was ligated together with the 1140 bp SalI-Xba I fragment of hPC:B148 cDNA into the pRC/CMV vector.

The cDNAs corresponding to WT hPC and the two variants QGNSEDY-hPC and QGNSEDY-hPC:B148, inserted in the eukaryotic expression vector pRc/CMV, were used to transfect the human kidney cell line 293. High-expressing colonies were selected for largescale culture and subsequent purification, as previously described [35]. The recombinant protein $\mathrm{C}$ variants were activated by thrombin, and then passed through a chromatography column of SP-Sephadex to remove thrombin essentially, as earlier described [36]. 


\section{In vitro coagulation analyses}

Citrated rat plasma was acquired from male Sprague-Dawley albino rats and used for in vitro characterization of the anticoagulant activities of the various hAPC variants. Increasing concentrations of the three hAPC variants were added in clotting assays using either the APTT reagent Platelin LS (Biomerieux, Marcy l'Etoile, France) or the PT reagent Simplastin Excel (Biomerieux). Each experiment was performed three times in duplicates and the mean values were used for presentation.

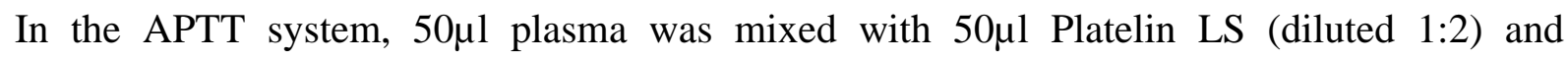
incubated for 180 seconds at temperature $37^{\circ} \mathrm{C}$ before initiation of coagulation by $50 \mu \mathrm{l}$ of $\mathrm{CaCl}_{2} 25 \mathrm{mM}$ with different concentrations of hAPC $(0,20,40,80$ and $160 \mathrm{nM})$. The concentrations of APC were chosen to correspond to the range of APC concentrations obtained after administration of the hAPC doses in the in vivo experiments. In the Simplastin (tisssue factor reagent) induced system, 50 $\mathrm{\mu l}$ plasma was incubated for 180 seconds at a temperature of $37^{\circ} \mathrm{C}$ before activation of coagulation by addition of $100 \mu$ l Simplastin Excel (diluted 1:50) containing increasing concentrations of the three forms of hAPC $(0,10,20,40$ and $80 \mathrm{nM}$ ). The dilutions of the reagents were made in Tris-Buffered Saline (TBS) $+0.1 \%$ Bovine Serum Albumin (BSA). The reason for diluting the reagents was that the clotting times were very short when undiluted reagents were used. The clotting times were measured using an Amelung KC 10 instrument (Amelung Heinrich, Lemgo, Germany).

\section{Thrombosis Model}

Fifteen Sprague-Dawley male albino rats with a mean body weight 291 grams (range 256-334 grams) were used in the pilot study. Fifty Sprague-Dawley male albino rats were used in the full-scale experiment with a mean body weight of 285 grams (range 246-334 grams). The experimental procedures were approved by the Research Ethics Committee at the University 
of Lund. Prior to the operations, the animals were housed in proper facilities with sufficient amounts of food, water and space. Each rat was anesthetized with a weight adjusted subcutaneous injection of a mixture of Hypnorm (fentanyl citrate $0.315 \mathrm{mg} / \mathrm{ml}$ and fluanisone 10 mg/ml, Janssen Pharmaceutica, Beerse, Belgium) and Dormicum (midazolam 5 mg/ml, F.Hoffman-La Roche AG, Basel, Switzerland) and sterile water. Thereafter, half of the initial dose was administered every 45 minutes by the same route. The right femoral vein was dissected free and cannulated with a polyethylene tubing (PE50; Intramedic, Parsippany, N.J, U.S.A) used for infusion of randomized substances and blood sampling. The left common carotid artery was dissected free from surrounding structures and isolated between two microvascular clamps (B-2V; S\&T Marketing, Neuhausen, Switzerland) resting on a soft plastic board in the wound. A short longitudinal incision was made in the vessel with a no.15 surgical blade (Swann-Morton, Sheffield, England) and a 7-mm longitudinal opening was completed with microscissors. The exposed vessel wall was everted and held in place on the soft plastic board with microneedles (Ethilon 10-0, BV-75-3; Ethicon Ltd, United Kingdom) put through the outer boarders of the vessel wall making the adventitia face flat down and the intima upward. A superficial transverse incision was made in the intima just distal to the two proximal needles and another incision just proximal to the distal needles to define the borders of the injury zone. By peeling off the inner vascular layers with two pairs of microforceps, a 5 mm long endarterectomy was performed, thus exposing adventitia or deep media, as described [37]. The anchoring needles were then removed. The arteriotomy was closed with a running Ethilon 10-0 monofilament polyamide suture (Ethilon 10-0, BV75-3) with approximately 30 suture loops. The clamps were then removed and the vessel reperfused. All blood emerging from the suture line was collected in preweighed cylindrical swabs (Dental Rolls No.1; IVF Schaffhausen, Neuhausen, Switzerland) dimensioned to fill the operating cavity. All swabs were collected in test tubes with caps to avoid evaporation. Weighing the 
swabs assessed the total blood loss from the arteriotomy. Thirty-one minutes after reperfusion, vessel patency was evaluated by the use of a standard microvascular empty-refill test [38] performed as follows. The vessel was occluded distal to the injury zone with a pair of microforceps, and emptied further distally with another pair of forceps. The first pair of forceps was released, and the vessel was classed as patent (rapid refilling) or occluded (no refilling). After completion of the experiment, the animal was given a lethal dose of a mixture of pentobarbital and alcohol. All operations were performed by one surgeon (K.M.). The mean total time for each experiment was 100 minutes (range 88-114) in the pilot study, and 102 minutes (range 91-122) in the full-scale experiment, from start of anesthesia to the time the animals were killed. A Leica Wild M650 microscope (Leica Microsystems, Wetzlar, Germany) was used for all microsurgical procedures, using magnifications x 16 and x 25.

\section{Experimental Protocol}

In the pilot study, 15 rats were divided into 5 groups and treated either with increasing doses of QGNSEDY-hAPC:B148 (0.1, 0.2, 0.4 or $0.8 \mathrm{mg} / \mathrm{kg})$ or vehicle only ( $\mathrm{n}=3$ in each group). In the full-scale experiment, 50 rats were divided into 4 groups that were treated either with recombinant WT hAPC $0.8 \mathrm{mg} / \mathrm{kg}$ (n=10), QGNSEDY-hAPC $0.8 \mathrm{mg} / \mathrm{kg}$ (n=10), QGNSEDYhAPC:B148 $0.8 \mathrm{mg} / \mathrm{kg}(\mathrm{n}=10)$ or vehicle only $(\mathrm{n}=20)$.

All experiments were performed in a blind random fashion. The randomized substances were stored in $1.5 \mathrm{ml}$ coded Eppendorf plastic tubes (Sarstedt, Numbrecht, Germany) at $-78^{\circ} \mathrm{C}$ and thawed to room temperature immediately before use. The substances were delivered as intravenous weight-adjusted bolus injections after completing the vascular injury, one minute before reperfusion. The infusion volume was $0.21 \mathrm{ml}$ per 100 -gram animal weight in all groups. The vehicle was $20 \mathrm{mM}$ Tris- $\mathrm{HCl}, 0.15 \mathrm{M} \mathrm{NaCl}$, $\mathrm{pH}$ 7.5, containing $2 \mathrm{mg} / \mathrm{ml} \mathrm{BSA}$. 


\section{Ex vivo coagulation analyses}

Blood samples $(0.9 \mathrm{ml})$ for coagulation analyses were drawn from the femoral vein a) 1 minute before injection of the randomized substance (baseline data), b) 2 minutes after reperfusion (i.e., 3 minutes after injection of the randomized substance) and c) 30 minutes after reperfusion (i.e., 31 minutes after injection of randomized substance and 1 minute before the patency test). Before each blood sample was collected, $0.5 \mathrm{ml}$ of blood was drawn into a separate syringe and wasted. After every $0.9-\mathrm{ml}$ sample of blood was collected, $0.5 \mathrm{ml}$ of saline was injected with another syringe to clear the catheter from blood.

The blood samples were collected with a 2-ml plastic syringe (Plastipak; Becton Dickinson AB, Hägersten, Stockholm, Sweden) prefilled with $0.1 \mathrm{ml}$ of $3.8 \% 0.13 \mathrm{M}$ sodium citrate. The samples were immediately transferred to 1.5-ml Eppendorf plastic tubes that were centrifuged at 14000 rpm for 3 minutes (Mikro 12/24; Hettich Zentrifugen, Tuttlingen, Germany). The plasma was separated with a pipette and put in two Eppendorf tubes with $300 \mu \mathrm{l}$ of plasma in each. The tubes were put in a box containing pellets of carbon acid ice. After completion of the daily experiments, the plasma samples were transferred to a freezer maintaining a temperature of $-78^{\circ} \mathrm{C}$.

The clotting time was analyzed with the APTT reagent Platelin LS (Biomerieux) diluted 1:2 in TBS + 0.1\% BSA and the PT reagent Simplastin Excel (Biomerieux) diluted 1:50 in TBS + $0.1 \% \mathrm{BSA}+10 \mathrm{mM} \mathrm{CaCl}$. The clotting times were measured using an Amelung $\mathrm{KC} 10$ instrument (Amelung Heinrich).

\section{Statistical Methods}

Patency rates were compared using Fisher's exact test and bleeding weights with the MannWhitney test. Statistics were calculated using StatXact 4.0.1 software (Cytel Software Corp., Cambridge, Mass., U.S.A.). Two-sided p-values are presented and p-values $<0.05$ were 
considered statistically significant. No correction was made for multiple testing. No statistical calculations were performed in the pilot studies since there were only three animals in each group 


\section{RESULTS}

\section{Expression and purification of recombinant protein $C$ variants}

The expression levels of the three recombinant proteins in 293-cells were between 3-5 mg/L. The proteins were collected under serum-free conditions and purified by anion exchange chromatography with overall recoveries of 35 to $40 \%$. On $10 \%$ SDS-PAGE the mutant and WT proteins migrated to similar positions corresponding to a molecular weight (MW) of approximately $60 \mathrm{kDa}$ (Fig. 1). The light and heavy chains of the proteins were separated after reduction of disulfide bonds. In the reduced forms, the $\alpha$ and $\beta$ (but no $\gamma$ )-isoforms of the heavy chain as well as the light chain were seen. All three reduced protein $\mathrm{C}$ variants contained a smaller amount of the single-chain form that disappeared after activation by thrombin, while the heavy chain isoforms shifted to slightly lower MW-positions, consistent with full activation (Fig. 1).

\section{In vitro anticoagulant effect}

In the in vitro clotting experiments, analyses with the APTT reagent Platelin LS (intrinsic pathway) showed that WT hAPC alone only slightly prolonged the clotting time in rat plasma. In contrast, the two mutants clearly prolonged the clotting time in a dose dependent manner with approximately tripled and quadrupled clotting times, respectively, at the highest concentrations (Fig. 2A). Analyses with the tissue factor pathway reagent Simplastin Excel showed similar clotting time patterns in rat plasma with pronounced anticoagulant effect achieved by the mutants and only a minor effect by WT hAPC (Fig. 2B). 


\section{Antithrombotic effect}

In the pilot study, $2 / 3$ vessels were patent in both groups given the highest doses $(0.4$ and 0.8 $\mathrm{mg} / \mathrm{kg}$ ) of QGNSEDY-hAPC:B148, 0/3 vessels in each of the groups receiving 0.1 and 0.2 $\mathrm{mg} / \mathrm{kg}$, and $1 / 3$ vessels in the control group (Fig. 3A). In the full scale experiment, the administration of WT hAPC or mutants did not produce any antithrombotic effect when compared to the control group, the patency rates being 3/10 vessels in the groups given WT hAPC and QGNSEDY-hAPC:B148 and 5/10 in the QGNSEDY-hAPC group, compared to $6 / 20$ vessels in the control group ( $p=1.00$ and $p=0.42$ respectively) (Fig. $4 \mathrm{~A}$ ).

\section{Arteriotomy bleeding}

In the pilot study, the arteriotomy bleeding seemed to be similar in all groups, but the groups were too small to make reliable statistical comparisons (data not shown). In the full-scale experiment, administration of different forms of hAPC did not increase the arteriotomy bleeding compared to controls; the median cumulative arteriotomy bleeding during 31 minutes of observation was 1.1 grams in the control group, which was not significantly different from the other groups (Fig. 4B).

\section{Ex vivo coagulation analyses}

In the pilot study, the clotting time increased in a dose dependent manner with QGNSEDYhAPC:B148 (Fig. 3B), and the clotting times in the highest dose were similar to those in the full scale experiment with the same mutant as described below. In the full-scale study, administration of WT hAPC only slightly increased the APTT in rat plasma. The mutant QGNSEDY-hAPC doubled and QGNSEDY-hAPC:B148 approximately tripled the clotting time, measured at three minutes after injection of substance (Fig. 4C). The clotting times measured by Simplastin Excel were increased by approximately 1.5 and 2 times, respectively, 
at three minutes after injection (Fig. 4D). At 31 minutes after injection, the clotting times had almost returned to baseline levels in all groups by both measurement methods. 


\section{DISCUSSION}

Arterial and venous thrombosis is a major medical problem motivating the search for improved antithrombotic agents. The specific anticoagulant activity of APC makes it an attractive candidate as a therapeutic compound and it has already proven its effect in association with severe septic shock [4, 17]. In models of arterial thrombosis in the rat, we have previously demonstrated bovine APC to be efficient as an antithrombotic agent when coadministered with bovine protein S [18-20, 32]. The present in vitro clotting experiments in rat plasma performed with either APTT or PT reagents, showed distinct dose dependent anticoagulant effect, especially with the mutant QGNSEDY-hAPC:B148 with four-fold prolongation at the highest concentration in the APTT system in contrast to WT-hAPC that only slightly prolonged the clotting time. The powerful anticoagulant response of the mutants generated the hypothesis that the mutants could be antithrombotic without co-administration of human protein S (hPS) in our experimental model. In the pilot study with increasing doses of the mutant QGNSEDY-hAPC:B148 there was antithrombotic effect in 4 out of 6 vessels in the two highest doses and the ex vivo anticoagulant response was similarly dose dependent as in the in vitro experiments. This combination of results led us to perform the larger study in which we observed the expected prolongation of the clotting time with the mutants but no significant antithrombotic effect compared to the control group, even though the pilot study had indicated that this could be possible.

The poor antithrombotic effects of hAPC now observed differ from observations in other studies, which report antithrombotic effects in arterial models of thrombosis in rats after intravenous injection of plasma purified hAPC. Smirnov et al 1990 demonstrated that injection of low doses of hAPC (15 or $30 \mu \mathrm{g} / \mathrm{kg}$ ) prolonged the time to complete occlusion of rat abdominal aorta that was made thrombogenic by electrical current [27]. Araki et al 1991 
showed that intravenous injection of hAPC $(0.9$ or $3.0 \mathrm{mg} / \mathrm{kg})$ significantly decreased the number of thrombotic occlusions and the total time the vessels were occluded in rat mesenteric artery after compression injury [25]. Yamashita et al 1994 described a rat model with repeated laser irradiation of mesenteric microvessels where intravenous injection of hAPC $(0.40 \mathrm{mg} / \mathrm{kg})$ significantly increased the number of laser irradiations necessary to achieve total occlusion of arterioles [28]. Hashimoto and colleagues showed decreased thrombus size after injection of hAPC $(1.62 \mathrm{mg} / \mathrm{kg})$ in rats with laser-induced thrombosis in mesenteric arterioles [29, 30]. The discrepancy between our and these previous findings may in part be explained by differences in the type of vascular injury used. In contrast to the previous studies [25, 27-30], the vascular trauma in our model was designed to mimic conditions that may be encountered in clinical settings, such as avulsion trauma or exposure of deep vascular layers in diseased vessels after atherosclerotic plaque rupture, i.e. a crude vascular trauma that produces occlusive thrombus formation and an injury that is prone to be relatively resistant to therapeutic intervention. Also, it is notable that the above-mentioned studies contained small treatment groups and were not performed in a blind randomized manner.

We have created several recombinant hAPC variants that express enhanced anticoagulant and catalytic effect in vitro as compared to WT hAPC and the aim of the present investigation was to test their efficacy as antithrombotic agents in vivo. The two hAPC variants we now used, QGNSEDY-hAPC and QGNSEDY-hAPC:B148 share the mutated Gla-domain, which provides the molecules with enhanced affinity for negatively charged phospholipids membranes. In a human plasma system, the QGNSEDY-hAPC variant has been shown to be highly efficient as anticoagulant [33] and in the in vitro clotting assay using rat plasma, this variant was much more potent as anticoagulant than WT hAPC. 
The QGNSEDY-hAPC:B148 variant, which combines the Gla-domain mutation with a loop 148 change that we previously found to be associated with enhanced catalytic and anticoagulant activity [34], was even more active than the QGNSEDY-hAPC variant in the in vitro clotting assays.

Despite being efficient as anticoagulants in vitro and ex vivo, none of the hAPC variants demonstrated antithrombotic effects in the rat model. It remains to be investigated whether the addition of human protein S make the hAPC variants more antithrombotic. With respect to the poor antithrombotic effect of the human APC variants, it should be remembered that the antithrombotic effects of the protein $\mathrm{C}$ system appear to be particularly important to inhibit thrombus formation on the venous side, as individuals carrying genetic defects affecting the system mainly suffer from venous and not arterial thrombosis [13, 14]. Thus, the APC variants we now investigated may be efficient in less thrombogenic settings and in models of venous thrombosis. As human APC is poorly active as an anticoagulant in rat plasma, it is also possible that these APC variants would be efficient as antithrombotic agents in humans.

In summary, we have investigated WT hAPC and two hAPC variants expressing increased anticoagulant activity in a rat arterial thrombosis model. None of the tested variants produced antithrombotic effect even though the two variant hAPCs gave distinct anticoagulant responses in rat plasma. 


\section{ACKNOWLEDGEMENTS}

We thank Mrs. Astra Anderson at the Department of Clinical Chemistry, University Hospital, Malmö for technical assistance. We also thank Professor Ulf Strömberg, Dept. of Occupational and Environmental Medicine, Lund University, for help with the statistical analyses. This work was supported by grants from the Swedish Science Council (\# 07143), the Österlund's Foundation, the Påhlsson's Foundation and by research funds from the University Hospital, Malmö (B.D., B.A.). 


\section{REFERENCES}

1. Dahlback B. Blood coagulation. Lancet 2000; 355:1627-32.

2. Dahlback B, Stenflo J. The Protein C Anticoagulant System. In: Stamatoyannopoulos G, Majerus P, Perlmutter R, Varmus H, eds: The Molecular Basis of Blood Diseases. WB Saunders; 2001. p. 614-656.

3. Esmon C. The protein C pathway. Crit Care Med 2000; 28:S44-8.

4. Esmon CT. The protein C pathway. Chest 2003; 124:26S-32S.

5. Nicolaes GA, Dahlback B. Factor V and thrombotic disease: description of a janusfaced protein. Arterioscler Thromb Vasc Biol 2002; 22:530-8.

6. Mann KG, Kalafatis M. Factor V: a combination of Dr Jekyll and Mr Hyde. Blood 2003; 101:20-30.

7. Kalafatis M, Rand MD, Mann KG. The mechanism of inactivation of human factor V and human factor Va by activated protein C. J Biol Chem 1994; 269:31869-80.

8. Nicolaes GA, Tans G, Thomassen MC, Hemker HC, Pabinger I, Varadi K, et al. Peptide bond cleavages and loss of functional activity during inactivation of factor $\mathrm{Va}$ and factor VaR506Q by activated protein C. J Biol Chem 1995; 270:21158-66.

9. Rosing J, Hoekema L, Nicolaes GA, Thomassen MC, Hemker HC, Varadi K, et al. Effects of protein S and factor Xa on peptide bond cleavages during inactivation of factor Va and factor VaR506Q by activated protein C. J Biol Chem 1995; 270:278528.

10. Mann KG, Hockin MF, Begin KJ, Kalafatis M. Activated protein C cleavage of factor Va leads to dissociation of the A2 domain. J Biol Chem 1997; 272:20678-83. 
11. Gale AJ, Heeb MJ, Griffin JH. The autolysis loop of activated protein C interacts with factor Va and differentiates between the Arg506 and Arg306 cleavage sites. Blood 2000; 96:585-93.

12. Norstrom EA, Steen M, Tran S, Dahlback B. Importance of protein S and phospholipid for activated protein C-mediated cleavages in factor Va. J Biol Chem 2003; 278:24904-11.

13. Lane DA, Mannucci PM, Bauer KA, Bertina RM, Bochkov NP, Boulyjenkov V, et al. Inherited thrombophilia: Part 1. Thromb Haemost 1996; 76:651-62.

14. Lane DA, Mannucci PM, Bauer KA, Bertina RM, Bochkov NP, Boulyjenkov V, et al. Inherited thrombophilia: Part 2. Thromb Haemost 1996; 76:824-34.

15. Esmon CT. Coagulation and inflammation. J Endotoxin Res 2003; 9:192-8.

16. Ruf W, Dorfleutner A, Riewald M. Specificity of coagulation factor signaling. J Thromb Haemost 2003; 1:1495-503.

17. Bernard GR. Drotrecogin alfa (activated) (recombinant human activated protein C) for the treatment of severe sepsis. Crit Care Med 2003; 31:S85-93.

18. Arnljots B, Bergqvist D, Dahlback B. Inhibition of microarterial thrombosis by activated protein C in a rabbit model. Thromb Haemost 1994; 72:415-20.

19. Arnljots B, Dahlback B. Protein S as an in vivo cofactor to activated protein C in prevention of microarterial thrombosis in rabbits. J Clin Invest 1995; 95:1987-93.

20. Arnljots B, Dahlback B. Antithrombotic effects of activated protein C and protein S in a rabbit model of microarterial thrombosis. Arterioscler Thromb Vasc Biol 1995; 15:937-41.

21. Jackson CV, Bailey BD, Shetler TJ. Pharmacological profile of recombinant, human activated protein C (LY203638) in a canine model of coronary artery thrombosis. J Pharmacol Exp Ther 2000; 295:967-71. 
22. Foo RS, Gershlick AH, Hogrefe K, Baron JH, Johnston TW, Hussey AJ, et al. Inhibition of platelet thrombosis using an activated protein C-loaded stent: in vitro and in vivo results. Thromb Haemost 2000; 83:496-502.

23. Gruber A, Griffin JH, Harker LA, Hanson SR. Inhibition of platelet-dependent thrombus formation by human activated protein C in a primate model. Blood 1989; 73:639-42.

24. Gruber A, Hanson SR, Kelly AB, Yan BS, Bang N, Griffin JH, et al. Inhibition of thrombus formation by activated recombinant protein $\mathrm{C}$ in a primate model of arterial thrombosis. Circulation 1990; 82:578-85.

25. Araki H, Nishi K, Ishihara N, Okajima K. Inhibitory effects of activated protein C and heparin on thrombotic arterial occlusion in rat mesenteric arteries. Thromb Res 1991; 62:209-16.

26. Sakamoto T, Ogawa H, Yasue H, Oda Y, Kitajima S, Tsumoto K, et al. Prevention of arterial reocclusion after thrombolysis with activated protein C. Comparison with heparin in a canine model of coronary artery thrombosis. Circulation 1994; 90:427-32.

27. Smirnov MD, Pyzh MV, Borovikov DV, Atorozhilova AN, Dobrovolsky AB, Golubych VL, et al. Low doses of activated protein C delay arterial thrombosis in rats. Thromb Res 1990; 57:645-50.

28. Yamashita T, Matsuoka A, Funatsu A, Yamamoto J. The antithrombotic effect of human activated protein $\mathrm{C}$ on He-Ne laser- induced thrombosis in rat mesenteric microvessels. Thromb Res 1994; 75:33-40.

29. Hashimoto M, Watanabe S, Oiwa K, Ohta Y, Kishi T, Okamoto T, et al. Enhanced thrombolysis induced by argatroban or activated protein $C$ in the presence or absence of staphylokinase, measured in an in vivo animal model using mesenteric arterioles. Haemostasis 2001; 31:80-9. 
30. Hashimoto M, Yamashita T, Oiwa K, Watanabe S, Giddings JC, Yamamoto J. Enhancement of endogenous plasminogen activator-induced thrombolysis by argatroban and APC and its control by TAFI, measured in an arterial thrombolysis model in vivo using rat mesenteric arterioles. Thromb Haemost 2002; 87:110-3.

31. Shibata M, Kumar SR, Amar A, Fernandez JA, Hofman F, Griffin JH, et al. Antiinflammatory, antithrombotic, and neuroprotective effects of activated protein $\mathrm{C}$ in a murine model of focal ischemic stroke. Circulation 2001; 103:1799-805.

32. Malm K, Dahlbäck B, Arnljots B. Prevention of thrombosis following deep arterial injury in rats by bovine activated protein $\mathrm{C}$ requiring co-administration of bovine protein S. Thromb Haemost 2003; 90:227-34.

33. Sun YH, Shen L, Dahlback B. Gla domain-mutated human protein C exhibiting enhanced anticoagulant activity and increased phospholipid binding. Blood 2003; 101:2277-84.

34. Shen L, Villoutreix BO, Dahlback B. Interspecies loop grafting in the protease domain of human protein $\mathrm{C}$ yielding enhanced catalytic and anticoagulant activity. Thromb Haemost 1999; 82:1078-87.

35. Shen L, Shah AM, Dahlback B, Nelsestuen GL. Enhancing the activity of protein C by mutagenesis to improve the membrane-binding site: studies related to proline-10. Biochemistry 1997; 36:16025-31.

36. Shen L, Shah AM, Dahlback B, Nelsestuen GL. Enhancement of human protein C function by site-directed mutagenesis of the gamma-carboxyglutamic acid domain. $\mathrm{J}$ Biol Chem 1998; 273:31086-91.

37. Soderstrom T, Hedner U, Arnljots B. Active site-inactivated factor VIIa prevents thrombosis without increased surgical bleeding: topical and intravenous administration in a rat model of deep arterial injury. J Vasc Surg 2001; 33:1072-9. 
38. Acland RD. Microsurgery Practice Manual. St. Louis: Mosby; 1980. p. 58-59 


\section{LEGENDS TO FIGURES}

Fig. 1 SDS-PAGE analysis of recombinant protein $\mathrm{C}$ variants. The purified proteins were subjected to $10 \%$ SDS-PAGE before and after activation by thrombin under both nonreducing (A) and reducing conditions (B). One microgram of protein was applied to each lane and the proteins were visualized by silver staining. The protein presented in each lane is noted below respective lane. The “Standard” contains the molecular weight markers. Activated proteins are marked in bold text. SC indicates single chain; HC, heavy chain; LC, light chain. The locations of the $\alpha$ and $\beta$ iso-forms of the HC are indicated.

Fig. 2 In vitro anticoagulant response in rat plasma produced by different concentrations of WT hAPC, or the two mutants of hAPC, measured with either the APTT reagent Platelin LS (A) or with the tissue-factor reagent Simplastin Excel (B). Each experiment was performed three times with measurements in duplicate. Mean values are presented. The indicated concentrations of hAPC refer to the final concentrations calculated, including all added volumes of reagents and hAPC as detailed in the Method section. 
Fig. 3 Effects of increasing doses of plasma purified hAPC or the mutant QGNSEDYhAPC:B148 in the rat model of arterial thrombosis in the blind randomized pilot study with three animals in each treatment group. Dotted bars represent the number of animals in each group, and the shaded parts of the bars indicate the number of patent vessels within each group.

A. Antithrombotic effect of QGNSEDY-hAPC:B148.

B. Ex vivo anticoagulant response (means) in rat plasma after intravenous injection of QGNSEDY-hAPC:B148, measured with the APTT reagent Platelin LS.

Fig. 4 Effects of WT hAPC and the two hAPC variants in the rat model of arterial thrombosis. ( $n=10$ in all treatment groups, $n=20$ in the control group).

A. Antithrombotic effect, expressed as vessel patency rates in percent. None of the hAPC variants produced any significant antithrombotic effect B. Arteriotomy bleeding (grams). All observations are plotted, as well as the $25^{\text {th }}, 50^{\text {th }}$ (medians) and $75^{\text {th }}$ percentiles (within boxes). The arteriotomy bleeding could not be statistically differentiated between the groups.

C and D. Ex vivo anticoagulant response in rat plasma (medians), measured with either the APTT reagent Platelin LS (C) or with the tissue-factor reagent Simplastin Excel (D). 


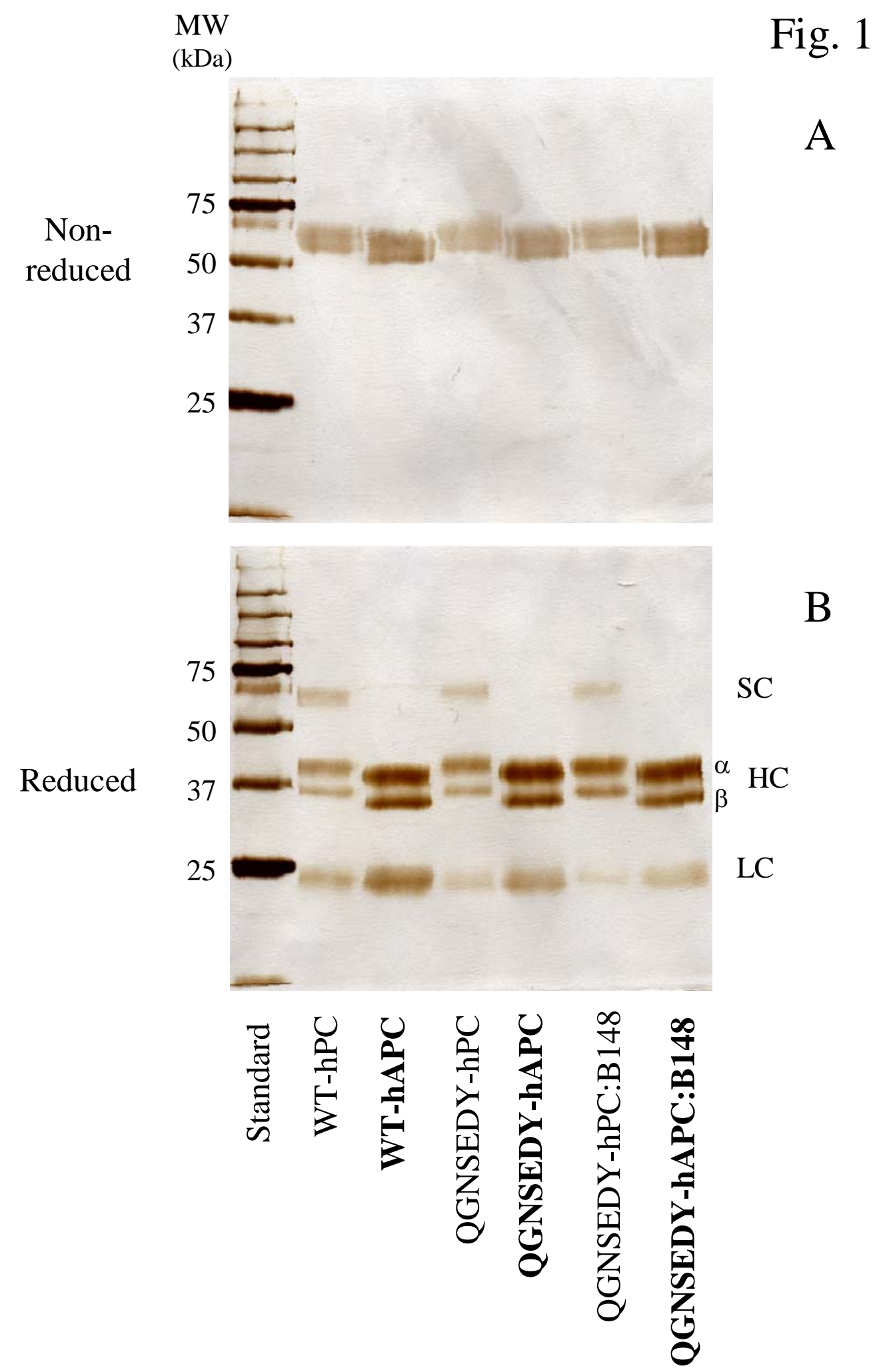


Fig. 2
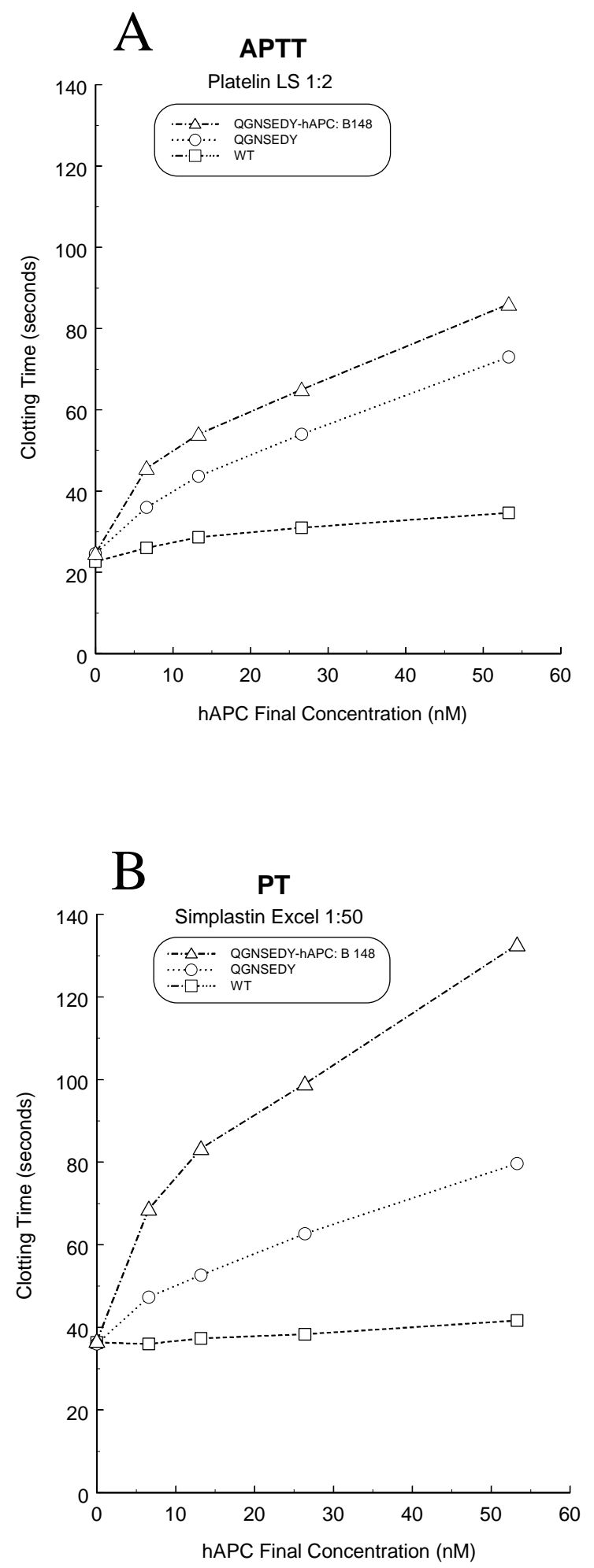
Fig. 3

A
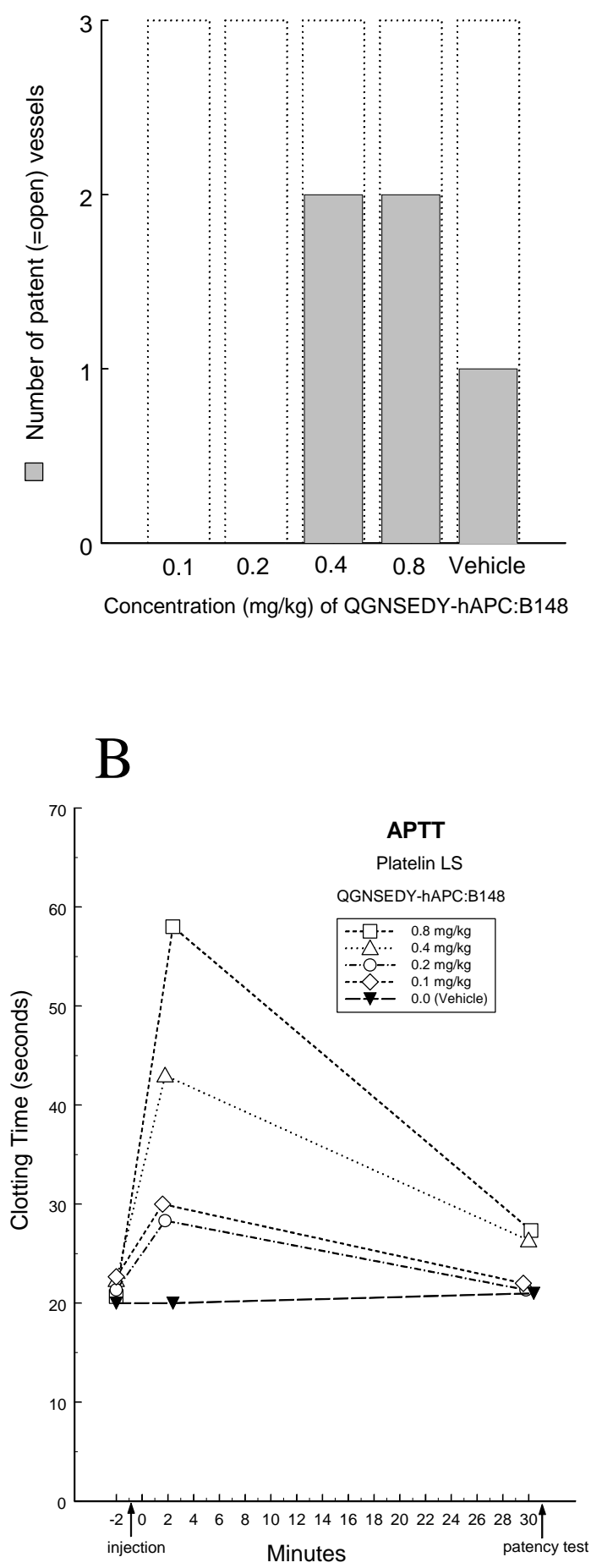
Fig. 4
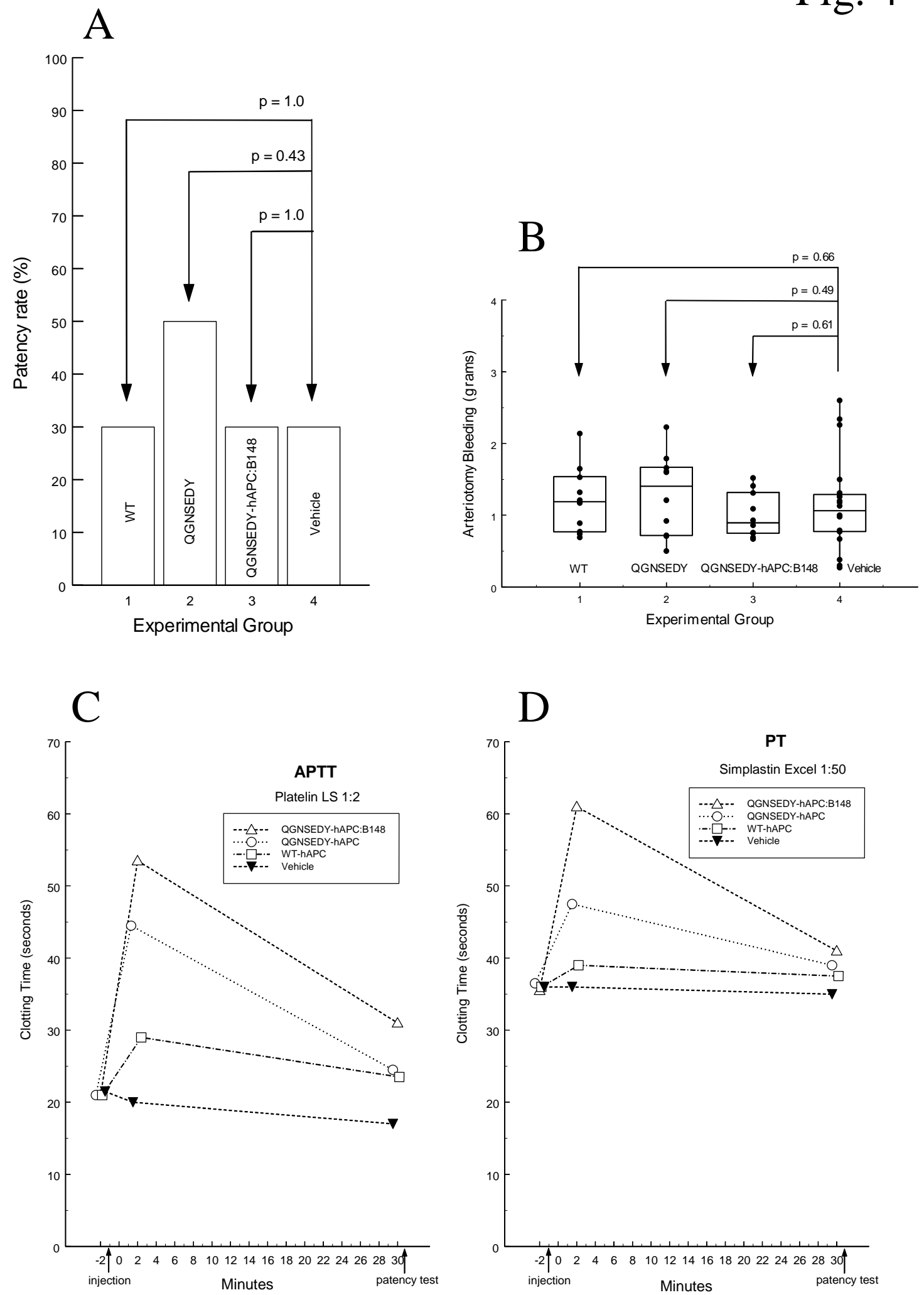